\title{
On Essential Incompleteness of Hertz's Experiments on Propagation of Electromagnetic Interactions
}

\author{
R. Smirnov-Rueda \\ Applied Mathematics Department, \\ Faculty of Mathematics \\ Complutense University, \\ 28040 Madrid, Spain
}

\begin{abstract}
The historical background of the 19th century electromagnetic theory is revisited from the standpoint of the opposition between alternative approaches in respect to the problem of interactions. The 19th century electrodynamics became the battlefield of a paramount importance to test existing conceptions of interactions. Hertz's experiments were designed to bring a solid experimental evidence in favor of one of them. The modern scientific method applied to analyze Hertz's experimental approach as well as the analysis of his laboratory notes, dairy and private letters show that Hertz's "crucial" experiments cannot be considered as conclusive at many points as it is generally implied. We found that alternative Helmholtz's electrodynamics did not contradict any of Hertz's experimental observations of transverse components as Maxwell's theory predicted. Moreover, as we now know from recently published Hertz's dairy and private notes, his first experimental results indicated clearly on infinite rate of propagation. Nevertheless, Hertz's experiments provided no further explicit information on non-local longitudinal components which were such an essential feature of Helmholtz's theory. Necessary and sufficient conditions for a decisive choice on the adequate account of electromagnetic interactions are discussed from the position of modern scientific method.
\end{abstract}

Key words: Hertz's experiments, velocity of propagation of interactions, longitudinal components, Helmholtz's electrodynamics, non-locality, action-at-a-distance

\section{Introduction}

Throughout the history of science, one can trace the importance of the notion of interaction in contrasting rival physical theories. Explanation of how remote particles influence each other through the empty space, challenged inquisitive minds of nearly all outstanding thinkers of earth civilization. In practice, we always observe one object acting on another by contact or at a distance. Before Newton introduced his revolutionary universal gravitation theory, the realist natural philosophy deriving from Aristotle considered only contact action. In its way to the practical knowledge the medieval science were laying down a new scientific method, breaking old religious prejudices and relying only on the simplest and clearest propositions. Postulation of undetectable, hidden or invisible causes responsible for interaction between material objects was inconceivable. 
The obvious break of that consensus came with the Newton's discovery of universal gravitation as an action-at-a-distance theory. It won immediate acceptance in England but not on the Continent, where the Cartesians regarded the notion of newtonian gravitation as an occult immaterial influence. This controversy took place at the time of the first scientific revolution when new conceptions were arising and changing very rapidly, hampered sometimes by confusions and prejudices. The arguments which lead medieval scholars to accept one or another theory were not only empirical or theoretical arguments. They also aroused partly out of the logical requirements of a particular metaphysics. Matter cannot act where it is not defined the cartesian metaphysical prohibition against action-at-a-distance. Types of scientific explanation began dependent upon the model of nature, i.e. could have been made precise only in the context of analogies or presupposed models.

The obvious success of Newton's theory in astronomy, similarity of electric and magnetic phenomena to gravitation confirmed later by Coulomb and Ampère, and the growing influence of empiricism in natural philosophy helped to modify the general consensus in favor of the direct action-at-a-distance. Mathematical models for hydrodynamics made it possible to describe the mechanism of transmission of influences through the continuous media. It was generally assumed that the gravitational attraction was transmitted instantaneously and, hence, always acted along the line joining the simultaneous position of two bodies. Bearing in mind the accuracy-limit of astronomical data at that time, Laplace ${ }^{(1)}$ in 1799 calculated that the velocity of propagation of gravitational interaction must have been at least eight orders of magnitude greater than that of light (modern discussion of this issue can be found in ${ }^{(2)}$ ). This respectable conclusion gave major support to the validity of the instantaneous action-at-a-distance (IAAAD) concept, leaving open the question of the physical cause of gravity. On this latter subject, a conceptual arm-wrestle was initiated between supporters and detractors of IAAAD. Newton himself had already thought that some physical mediator must exist. It is absurd, he said ${ }^{(3)}$, to suppose that gravity is innate and acts without a medium, either material or immaterial. Many eminent scientists like Laplace explained the phenomenon as due to an impulsion of some immaterial fluid, but did not find it appropriate at the time to search for a reliable physical explanation of the cause of interaction, and so they bequeathed this task to future generations. There is no need at all, Laplace ${ }^{(4)}$ declared in 1796, to posit vague causes, impossible to submit to analysis, and which the imagination modifies to its liking in order to explain these phenomena.

At that stage, it is not surprising that for many of the adversaries of IAAAD these suggestions of immateriality could not be dissociated from spiritual, religious and other non-scientific notions. This path of reasoning led them to conclude that if the IAAAD concept did not imply any material mediator, then it did not imply any physical mediator at all. Thus, in their opinion IAAAD became inconceivable from the point of view of common-sense logic. This prepared the ground for the reappearance of an alternative and half-forgotten Aristotelian concept, namely, that of action by local contact (or contact action $)^{(5)}$. In its modern form known as the local field concept, it was reintroduced by Faraday, giving apparently a more satisfactory physical description of the interaction phenomena. It was based on the causality of local interactions in terms of material 
forces filling space. After the discovery of electromagnetic induction, Faraday became convinced that electric and magnetic influences were propagated through a material medium rather than at a distance through an empty space.

Thus, nearly one and a half century after Newton's discovery of the action-at-adistance theory for gravitational attraction, the general consensus was broken again. It resulted in polarizing views on both sides of the Channel: paradoxically, the majority of English scholars were now on the side of Faraday's field conception, whereas the notion of the direct action-at-a-distance still remained fundamental on the Continent. Thus, the 19th century electrodynamics became the battle-field of a paramount importance to test existing conceptions of interaction. There is no doubt that Hertz's contribution was decisive in that historical choice. He was the first physicist who observed electromagnetic waves in air travelling with the velocity of light. Since then it is commonly believed that on the classical level the question of the general meaning and understanding of electromagnetic interactions and their mechanisms had been positively resolved.

In this work we will discuss that Hertz's experimental approach on propagation of electromagnetic interactions cannot be considered as conclusive at many points as it is generally implied and, hence, the central battle of the 19th century physics cannot been considered definitely won by one of the opposing sides. This conclusion depends essentially upon the correct reconstruction of the historical retrospective of the 19th century's electromagnetic research.

\section{Historical Retrospective of the 19TH Century's Electro- magnetism and its Conceptual Background}

In order to appreciate the difficulty and the importance of the task undertaken by Hertz in his experimental investigations, it is worth recalling the uncertain and highly controversial state of electrodynamics of the 19th century. Hertz himself was trained in the research tradition of the Berlin school headed by Helmholtz ${ }^{(6)-(7)}$, who from the middle of 1860's, had sought to clarify existing principles in electromagnetic theory and to reach a consensus between the two major directions in electromagnetic research of that time, namely, Newton's instantaneous action-at-a-distance concept as used by Weber, and Faraday's contact action concept as developed by Maxwell. By the time of Helmholtz's first attempt at reconciliation (1870), the theoretical schemes of Weber and Maxwell had successfully incorporated all previously well-established descriptions and empirical facts, such as the electric potential theory (electrostatics), Ampère's magnetostatics and Faraday's theory of induction.

Weber developed his theory (1848) in accordance with the Newtonian program, which prescribed that all forces between pairs of particles should be radial, acting directly through space (i.e. along the line joining particles) without any observable material mediator. Restriction on this radial description of electromagnetic forces came from Ampère, who understood that instantaneity meant no delay, hence no aberration. Any aberration attending the finite propagational velocity of interactions would imply nonradial forces. However, the existence of radial forces as a basic assumption of instanta- 
neous action-at-a-distance (IAAAD) theories was confirmed by Ampère experimentally to the degree of accuracy available at that time. Thus, electric and magnetic interactions were thought to be complete analogy to gravitational attractions which, according to astronomical observations, always acted along the line joining simultaneous positions of two bodies.

At first time, Faraday's material field concept attracted many scholars seeking rather for the physical explanation than for the purely mathematical description of gravitational and electromagnetic phenomena. But later on, the perspective opened by Faraday seemed to be so wide and novel that it challenged to explore it deeply many excellent mathematicians of that time and among them Maxwell, who also tried to work out his own comprehensive field theory based on Faraday's concept. However, Maxwell himself was aware of the provisional status of this approximation and encountered some conceptual difficulties, since he had incorporated all the basic IAAAD results such as electrostatics and magnetostatics without any modification. With regard to the lines of force treated by Faraday as the representation of a material field, Maxwell's own position was still undefined, but he cautiously dealt with them as if they were lines of flow of an incompressible, imaginary fluid. As Maxwell stated ${ }^{(8)}$ :

The substance here treated of must not be assumed to possess any of the properties of ordinary fluids except those of freedom of motion and resistance to compression. It is not even a hypothetical fluid which is introduced to explain actual phenomena. It is merely a collection of imaginary properties which may be employed for establishing certain theorems in pure mathematics in a way more intelligible to many minds and more applicable to physical problems than that in which algebraic symbols alone are used.

Consequently, being in a static limit, mathematically equivalent to older IAAAD theories, the status of contact field theories could not have been considered free of ambiguity (a fuller discussion of this can be found in M. Hesse work ${ }^{(9)}$ ). In other words, in this static limit, electric and magnetic fields behaved very much as flows of an ideal, incompressible fluid, in which case they were indistinguishable from IAAAD. These uncertainties in Maxwell's original theoretical scheme were later summarized clearly and concisely by Hertz, who wrote ${ }^{(10)}$ :

Maxwell's own representation does not indicate the highest attainable goal; it frequently wavers between the conceptions which Maxwell found in existence, and those at which he arrived. Maxwell starts with the assumption of direct action-at-a-distance; he investigates the laws according to which hypothetical polarizations of the dielectric ether vary under the influence of such distance-forces; and he ends by asserting that these polarizations do really vary in this way, but without being actually caused to do so by distanceforces. This procedure leaves behind it the unsatisfactory feeling that there must be something wrong about either the final result or the way which led to it. 
As an approach to clarifying these uncertainties, let us examine the essential distinction between the conceptual foundations of IAAAD and those of contact-field doctrines. At the beginning of the 19th century, the possibility of a final explanation of the mechanisms of IAAAD as the basis of gravitational and electric forces had not been completely ruled out. Moreover, due to the rapid development of theoretical hydrodynamics, the attitude towards IAAAD changed from the summary rejection of its unphysical status to an awareness of a deep similarity between the potential function and the velocity-field of a fluid. It had been realized that the main difference between IAAAD and Faraday's field was the fact that in IAAAD a potential need not necessarily describe a material property of anything, whereas for Faraday it was the property of a material substance which could be observed in ways familiar to ordinary matter such as, for instance, liquids and gases. Like any other material substance, therefore, Faraday's field could be regarded as something movable with positive kinetic energy and, therefore, detectable empirically. This suggests that an important criterion, as Hesse puts it ${ }^{(11)}$ :

... in deciding whether or not a field is to be regarded as a physically continuous medium rather than a mere mathematical device lies in its possession of detectable properties other than the one property for which it was introduced. A condition of this kind is often suggested as a of the physical 'reality' of a theoretical entity, and it led Faraday to express his dissatisfaction with Newtonian gravitation. But independent detection was not the only consideration which weighed with the nineteenth-century physicists. They were prepared to regard a field as a physically continuous medium on other and less stringent terms, for example, if propagation was affected by material changes in the intervening space, if it took time, if a mechanical model could be imagined for the action of a medium in producing the observed effect, or if energy could be located in the space between interacting bodies. Any of these three conditions might be regarded as sufficient and no one of them was individually necessary. Thus, gravitation remained an action at a distance throughout the nineteenth century, in spite of its description by a potential theory, because it did not satisfy any of these criteria, whereas the electromagnetic field theory began to take on the characteristics of continuous action because it satisfied all of them. It is sometimes suggested in modern (post-relativity) works that a finite velocity of propagation is a necessary as well as sufficient condition for continuous action, and that this is why instantaneous gravitation could not be regarded as such an action, but in classical physics there is instantaneous transmission of pressure and of longitudinal waves in an incompressible medium, and this would certainly be regarded as continuous action.

From this preliminary survey of the two opposite conceptual foundations, we may conclude that the 19th century physicists considered as sufficient conditions for validity of Faraday-Maxwell's field concept:

1) propagation of fields producing material changes in the surrounding space; 
2) time-delay in propagation;

3) experimental observation of the energy transfer related to interactions;

Nevertheless, as it will be discussed later, the above-mentioned conditions should be considered only as necessary, not sufficient for establishing the existence of Faraday-type contact fields, since there might be a third alternative which would combine both IAAAD and Faraday's contact-field features in a single scheme. Formally, such a scheme (with superposition of material and immaterial substances) would satisfy all above mentioned conditions but on theoretical foundations different from those of purely material contactfield theory. In this respect, it will be shown when examining the historical background of the 19th century electrodynamics, that such a third alternative did actually exist and that this was the so-called compromise theory of Helmholtz. It means that the problem of the completeness of Hertz's crucial experiments should be again revisited but from methodological and philosophical positions of modern science.

\section{Status of Different Electrodynamics Doctrines Before Hertz's Experiments}

By the time Helmholtz became actively involved in resolving problems of electromagnetism, in the middle of the 1860's, Weber's and Maxwell's supporters had already been locked in a lengthy and futile conflict. Helmholtz attempted to make a decisive choice between them by constructing his own mathematical scheme and designing crucial experiments to weigh in favor of either Weber's or Maxwell's theory.

Helmholtz ${ }^{(12)}$ attempted to elaborate his compromise approach aimed at combining the important elements of the two theories. When trying to arrive at results similar to Maxwell's without losing the elements of action-at-a-distance, Helmholtz assumed that the electrostatic forces are constantly present as a field in space and that the change in the polarization or the displacement of the charges signalled the change in the electrostatic field. As discussed by Kudryavtzev ${ }^{(13)}$ and by Buchwald ${ }^{(14)}$, under these assumptions, Helmholtz successfully derived generalized equations very similar to those of Maxwell and found that in a limit case they yielded equations identical to Maxwell's equations for scalar and vector potentials. Solving these equations for a homogeneous dielectric medium, he arrived at the wave equations for electric and magnetic polarizations, respectively, with undetermined constant $k\left(\operatorname{see}^{(13)-(15)}\right)$.

The conciliatory aspect of Helmholtz's approach resulted in the following peculiarity. To reach formally Maxwell's scheme from Helmholtz's approach required two limits: $k=0$ and infinite ether susceptibility (for greater details see Appendix A). The former was necessary to obtain Maxwell's equations for potentials and the latter, to make the velocity of transverse waves in Helmholtz theory equal to the velocity of light $c$. In addition to the ordinary transverse electromagnetic waves already confirmed by Maxwell, Helmholtz discovered the existence of longitudinal electric waves which turned out to be instantaneous at the Maxwellian limit. Interpretation of this conclusion and its consequences became a hard nut to crack for all contemporary electrodynamicists. Maxwellian followers (Heaviside, FitzGerald, Lodge etc.) refused to accept Helmholtz's theory because 
they found his conceptions entirely foreign to Maxwell's view of the transmission of interaction. Helmholtz himself recognized conceptual differences between the two theories but insisted on their remarkable similarity ${ }^{(16)}$ :

...It follows. ...from these investigations that the remarkable analogy between the motion of electricity in a dielectric and that of the light ether does not depend on the particular form of Maxwell's equations hypotheses, but results also in a basically similar fashion if we maintain the older viewpoint about electrical action at a distance.

Helmholtz's attempt at a more consistent reformulation of the contemporary electrodynamics theories could not, however, resolve the problem of which approach to favor. His theory contained the undefined constant $k$ which could have any possible non-negative value and still remain compatible with existent experimental data, since most of it had been obtained for closed currents where the value of $k$ is irrelevant. Even for values of $k$ close to unity in the case of open currents, an experimental effort towards obtaining reliable results would require an enormous boost in accuracy and would therefore require new kinds of measurement devices. Despite the difficulties in carrying out any experimental task of this kind, Helmholtz, along with N. Schiller and H. Rowland, his pupils and followers at the University of Berlin, performed in 1874-78 a series of preliminary experiments on open currents. All results were in apparent agreement with the law of electromagnetic induction described equally well by the Neumann, Maxwell and Helmholtz theories. Therefore, the need for new decisive and reliable experimental data was still urgent when Hertz became interested in electromagnetic research.

\section{Hertz's Contribution to Electromagnetic Research}

In 1879 Helmholtz proposed a prize competition, " To establish experimentally a relation between electromagnetic action and the polarization of dielectrics" and urged his student Hertz to take up the challenge. At first, Hertz declined, discouraged by the poor prospects of success at that time. Later on, he began investigating the problem for his own interest. In 1886-88, at Karlsruhe, he attempted to establish the compatibility of the theories of Helmholtz and Maxwell in a new series of experiments. He designed his measurement-procedure, taking into account Helmholtz's separation of the total electric force into the electrostatic and electrodynamic parts to which different velocities of propagation were ascribed. In Hertz's words ${ }^{(17)}$ :

The total force may be split up into the electrostatic part and electrodynamic part; there is no doubt that at short distances the former, at great distances the latter, preponderates and settles the direction of the total force.

In spite of apparent difficulties, Hertz decided to carry out those measurements. For his purpose he had to develop new devices providing electrical oscillations faster than had been previously available. His efforts were, however, rewarded and he quickly 
demonstrated the existence of very rapidly varying currents with a strong inductive action across the discharge gap ${ }^{(18)}$. He also established a resonance-like relationship between the primary and secondary circuits in the presence of regular oscillations. As a result of these investigations, a solution to the Berlin Academy's problem could be considered possible.

The next important step was to modify and improve the detection apparatus (secondary circuit) in order to undertake interference experiments in which he used mutually orthogonal wire and oscillator. Hertz's idea was the following: the oscillator sends out a given phase along the wire as well as a given phase of direct action through air (which differs from the wire phase by a quarter of a period). If both phases travel at the same rate, their combined effect (interference) will be the same along the wire. Otherwise, the effect will change at different point.

At the end of 1887, On November 11, he finally undertook this task and observed the existence of the interference between the oscillations propagated in air and wire. The entry of his dairy notes for November 12 reads: "Set up experiments on the velocity of propagation of the electromagnetic effect. Contrary to expectations, the result is infinite propagation". In retrospective, Hertz gave account about these first experiments in the Introduction to his "Electric Waves":

...Dishearted, I gave up experimenting. Some weeks passed before I began again. I reflected that it would be quite as important to find out that electric force was propagated with an infinite velocity, and that Maxwell's theory was false, as it would be, on the other hand, to prove that this theory was correct, provided only that the result arrived at should be definite and certain...

As we know from his dairy, he decided to write a detailed paper on negative results but only after having checked and ensuring his previous results. Five weeks later, On December 15-22, Hertz returned to his experiments and confirmed apparently infinite propagation of direct action (On the sequence of Hertz's first experiments, see Appendix B).

On December 26 Hertz began a new series of experiments using a new position of the resonator. According to the measured interference phase shift, Hertz unexpectedly found that the oscillator's direct action propagated 1.5 times faster than the wire wave (i.e. close to the velocity of light). Setting the resonator in different positions Hertz obtained interference marks which he put in the final table. As we shall discuss below, these results contained a singularity: an apparently infinite rate of propagation in region very close to oscillator (near zone).

After some doubts (see Appendix B) Hertz compiled all result of the last experiments and published in a paper entitled "On the Finite Velocity of Propagation of Electromagnetic Action" (1888) which nowadays, according to the established historiography of physics, is considered a classical reference in which the difficult task of proving the finite propagation velocity of electromagnetic interactions in air had been achieved (it should be noted here that the title of this Hertz's paper is perhaps misleading nowadays, because conventional Maxwellian electrodynamics does not employ the Helmholtzian action ter- 
minology, nor does it split the total electric force into electromagnetic and electrostatic parts).

It is important to understand what Hertz had in mind when published this paper. As we know from Hertz's dairy and letters, he was eagerly looking for a positive result, i.e completely novel effects (see Appendix B). After the last series of experiments he became strongly convinced the electrodynamic action had a finite rate of propagation. However, he was not sure how to combine it with the previous experiments and found it possible to question their viability in the new light of novel "positive results". In fact, he admitted that his first experiments could not be considered reliable to conclude something definitive about longitudinal components. Looking carefully through the same paper, we find Hertz declaring to support his claims ${ }^{(19)}$ :

From this it follows that the absolute value of the first of these is of the same order as the velocity of light. Nothing can as yet be decided as to the propagation of electrostatic actions.

In later retrospective, writing the Introduction to his "Electric Waves" Hertz argued apparent implications of his "negative" results by inaccuracy in observations (for more details see Hertz's Introduction to "Electric Waves and also Appendix B).

Concerning the final table of interference marks which he published in the paper "On the Finite Velocity of Propagation of Electromagnetic Action" (1888), it should be emphasized that some of Hertz's measurements tended to manifest the instantaneous nature of the electrostatic mode, but he was also not convinced of this instantaneity and preferred to be cautious, since his method was unable to provide him with any reliable quantitative results ${ }^{(20)}$ :

Since the interferences undoubtedly change sign after 2.8 meters in the neighborhood of the primary oscillation, we might conclude that the electrostatic force which here predominates is propagated with infinite velocity. But this conclusion would in the main depend upon a single change of phase... If the absolute velocity of the electrostatic force remains for the present unknown, there may yet be adduced definite reasons for believing that the electrostatic and electromagnetic forces possess different velocities.

These circumstances of Hertz's experiments were previously noted by some researchers on history of physics such as Cazenobe ${ }^{(21)}$ and Doncel ${ }^{(22)}$. An excellent reconstruction of Hertz's "crucial" experiments in great detail was undertaken by Buchwald ${ }^{(14)}$ where one can appreciate some of Hertz's major doubts and hesitations (see Appendix B).

In spite of all difficulties and uncertainties of these first measurements, Hertz was fully aware of the need for additional experiments to cast some light of certainty on the electrostatic $\operatorname{part}^{(19)}$ :

It is certainly remarkable that the proof of a finite rate of propagation should have been first brought forward in the case of a force which diminishes in inverse proportion to the distance [electrodynamic part], and not to the 
square of the distance [electrostatic part]. But it is worth while pointing out that this proof must also affect such forces as are inversely proportional to the square of the distance. For we know that the ponderomotive attraction between currents and their magnetic actions are connected by the principle of the conservation of energy with their inductive actions in the strictest way, the relation being apparently that of action and reaction. If this relation is not merely a deceptive semblance, it is not easy to understand how the one action can be propagated with a finite and the other with an infinite velocity.

Hertz's point seems to tell his growing insatisfaction with the complexity of Helmholtz's theoretical approach. In his opinion, two different velocities ascribed in Helmholtz's theory to two different parts of action made the whole task of test and analysis unreasonably complicated. Hertz inclined towards justification of Faraday-Maxwell's field approach as a special limit of Helmholtz's theoretical scheme and also as based on a simpler model.

Hertz's conversion to Maxwellian ideas is well-discussed in some history and philosophy of science texts ${ }^{(23)}$. Nevertheless, it is interesting to follow briefly the evolution of Hertz's theoretical ideas. He started with Helmholtz's theory, and his conversion to Maxwell's viewpoint was an uneasy process, possibly never fully completed due to his (Hertz's) premature death (On January 1, 1894). He began analyzing the underlying concepts in the Maxwellian limit of Helmholtz's theory (see Appendix A) but his final interpretation became essentially different in form from what had been commonly accepted by Helmholtz and his followers. More specifically, Hertz uncritically assumed that in Maxwell's limit the instantaneous longitudinal component should have been excluded from consideration in Helmholtz's original theory. All forces then became explicitly time dependent, i.e. possessing the finite velocity of propagation.

This was a drastic departure from the Hertz mentor's position on the nature of electromagnetic interactions and, in general, from his philosophical foundations. In fact, Helmholtz rejected time dependent forces (he admitted only implicit time dependence upon space position or direct action-at-a-distance (IAAAD)) and was deeply convinced that ${ }^{(24)}$

...nature could only be comprehended through invariable causes. Helmholtz viewed electromagnetic interactions - indeed, all interactions, - as instantaneous and bipartite...

and, therefore, could attribute interactions only to longitudinal components (electrostatic action in Helmholtz's classification).

Let us see an aberration of Helmholtz's ideas in Hertz's own words ${ }^{(25)}$ :

...Helmholtz distinguishes between two forms of electrical force the electromagnetic and the electrostatic to which, until the contrary is proved by experience, two different velocities are attributed. An interpretation of the experiments from this point of view could certainly not be incorrect, but it might perhaps be unnecessarily complicated. In a special limiting case Helmholtz's theory becomes considerably simplified, and its equations in this case become 
the same as those of Maxwell's theory; only one form of the force remains, and this is propagated with the velocity of light. I had to try whether the experiments would not agree with these much simpler assumptions of Maxwell's theory. The attempt was successful. The result of the calculation are given in the paper on "The Forces of Electric Oscillations, treated according to Maxwell's Theory".

This paper was published in 1889, one year after the discussion of Hertz's first results, which apparently were not sufficient to conclude which of the two theoretical descriptions was more adequate. Hertz evaded the wide analysis of the nature and mechanisms of electromagnetic interactions, reducing it to the limited task of experimental verification of some predictions of Maxwell's theory. In this comprehensive paper Hertz tried to show how the observed singularities in the propagation of the electric force could be described by Maxwell's theoretical scheme. As Hertz explained ${ }^{(26)}$ :

The results of the experiments on rapid electric oscillations which I have carried out appear to me to confer upon Maxwell's theory a position of superiority to all others. Nevertheless, I based my first interpretation of these experiments upon the older views, seeking partly to explain phenomena as resulting from co-operation of electrostatic and electromagnetic forces. To Maxwell's theory in its pure development such a distinction is foreign. Hence I now wish to show that the phenomena can be explained in terms of Maxwell's theory without introducing this distinction. Should this attempt succeed, it will at the same time settle any question as to a separate propagation of electrostatic force, which is meaningless in Maxwell's theory.

In this famous paper, Hertz wrote Maxwell's equations in the form in which they are known today (the Hertz-Heaviside form) and also derived the distribution of force lines for the radiating oscillator (Hertz vibrator). In other words, this important contribution to the Faraday-Maxwell field theory consisted in the development of the general sourcefield relation previously unknown. (Today this method bears Hertz's name and is based on the straightforward Fourier analysis of dipole and multi-dipole radiation.) Using these calculations, Hertz found an explanation (alternative to that based exclusively on Helmholtz's ideas) of the singularities he had observed in the distribution of radiation in the near field (the apparently instantaneous behavior of the electrostatic component). In Hertz's words ${ }^{(27)}$ :

Let us now investigate whether the present [Maxwell's] theory leads to any explanation of the phenomena... At great distances the phase is smaller by the value $\pi$ than it would have been if the waves had proceeded with constant velocity from the origin; the waves, therefore, behave at great distances as if they had travelled through the first half wavelength with infinite velocity.

Interestingly, this prediction of Maxwell's theory concerning the infinite phase-velocity for the near-field zone based on straightforward Fourier analysis (Hertz's method) appears in modern texts ${ }^{(28)}$. Surprisingly, however, that Hertz himself paid no attention to 
this prediction beyond the fact that it gave him a new interpretation of his experimental results, different from that provided previously only by the Helmholtzian approach. It is possible that Hertz did not realize (or had no time to realize) all conceptual implications of the new prediction, which has no clear meaning in the framework of the Faraday-Maxwell contact-field doctrine. The prediction would imply the existence of a small, but macroscopic region where the notion of Faraday locality results invalid. On the other hand, it reproduces above-discussed fuzziness in the relationship between static and dynamic limits in Maxwell's theory.

Bearing that ambiguity in mind, it would not have been surprising if Maxwell's theoretical predictions for static and quasistatic phenomena had been found to be similar to the older IAAAD views. It is obvious that phenomena in the near field zone (less than half wavelength) should be regarded as quasi-static in the Hertzian analysis and therefore, implicit time dependent that is not Maxwellian but Helmholtzian feature for longitudinal components. This reasoning should have cast doubts on Hertz's explanation of the experimental results as not being completely in the spirit of the Faraday-Maxwell's conceptual foundations. It is surprising, then, that almost no-one seemed to have been worried by this presence of non-Faraday's elements in Maxwell's approach. By the same token, it is no less surprising that Hertz's explanation was so unconditionally accepted by Maxwell's followers.

Although Hertz was satisfied that his calculations had accounted for the majority of the observed phenomena, he stressed that he had not succeeded in removing all the difficulties from his experimental verification of Maxwell's theory. He confessed that ${ }^{(29)}$ :

I have therefore repeated the experiments, making various alterations in the position of the primary oscillator, and found that in certain positions the results were in accordance with theory. Nevertheless, the results were not free from ambiguity, for at great distances in places where the force was feeble, the disturbance due to the environment of the space at my disposal were so considerable that I could not arrive at a trustworthy decision.

This shows that some of Hertz's measurements had been made to the limit of accuracy available at the time. In his final experimental paper titled "On Mechanical Action of Electric Waves in Wires", published in 1891 (after his departure from Karlsruhe), Hertz attempted to observe the existence of magnetic waves accompanying the electric waves in order to disprove any reference to action-at-a-distance. As far as the problem of electromagnetic interaction is concerned, this last task was an important attempt on a definite experimental demarcation of irreconcilable views. However, he was forced to $\operatorname{admit}^{(30)}$ :

...I hoped to be able to devise some way of making observations on waves in free air, that is to say, in such a manner that any disturbances which might be observed could in no wise be referred to any action-at-a-distance. This last hope was frustrated by the feebleness of the effects produced under the circumstances. 
Additionally, there were no empirical indications of the energy transfer between interacting electrically charged bodies at rest. The question remained open whether Maxwell's transverse waves of interaction were too feeble to be detected or, otherwise, they had no right to exist according to Helmholtz's views. Thus, Hertz himself recognized that the fundamental question on the nature and mechanisms of interaction could not have been definitively clarified by experimental tools available at that time.

Although Hertz's satisfaction with Maxwell's theory was understandable, there were still insufficient number of arguments for making a truly decisive choice, bearing in mind that Helmholtz's approach remained in qualitative agreement with the observed singularities. However, no further experiments or calculations for testing the quantitative predictions of Helmholtz's theory have been attempted.

...The problem Hertz encountered with Helmholtz's theory was thus of a very special sort. It does not consist in a contradiction between an empirical observation and theory, nor does it consist in a contradiction among theoretical statements. Instead it consists in a philosophical problem with theoretical terms: what is their meaning, how do they acquire their meaning, how can they be rendered consistent. ${ }^{(31)}$

In sum, we can conclude that no empirical indication of the inadequacy of Helmholtz's theory had ever been observed or declared by Hertz, so that from the position of modern scientific method it is difficult to conceive why Helmholtz's theory had been ruled out as a possible alternative. In a mathematical language, we would say that Hertz found only necessary conditions to accept Maxwell's theory. If those conditions were sufficient as well, then Helmholtz's theory would have been discarded on a solid logical basis. In the next section, necessary and sufficient conditions for a decisive choice on the adequate account of electromagnetic phenomena and interactions will be discussed.

\section{Discussions}

In modern retrospective of the above-stated reservations of Hertz, as well as of some other thinkers of the time, the unconditional acceptance with which Hertz's experiments and their interpretations were received might seem somewhat unjustified. Hertz himself did not expect such support and attributed it to the heavy philosophical burden of the old and unresolved dilemma of the choice between the IAAAD and contact-action doctrines ${ }^{(32)}$ :

The approval with which they have been received has far exceeded my expectations. A considerable part of this approval was due to reasons of a philosophic nature. The old question as to the possibility and nature of forces acting at a distance was again raised. The preponderance of such forces in theory has long been sanctioned by ordinary common sense; in the domain of electricity these forces now appeared to be dethroned from their position by simple and striking experiments... The details of the experiments further prove that particular manner in which the electric force is propagated exhibits 
the closest analogy with the propagation of light; indeed, that it corresponds almost completely to it.

There is no doubt, then, that Hertz already considered the propagation of electric interaction as a transverse wave, completely analogous to the light-propagation, due to its being the only explicit indication of his experiments. Another detail which may also have contributed to the unconditional approval of Hertz's results among the scientific community was, as already stated, the rather misleading title of his paper on the finite propagation of electromagnetic actions, possibly due to unawareness of Helmholtz's classification of forces.

However, in contrast to this general enthusiasm for Hertz's results, there was also some strong opposition from P. Duhem, an eminent French mathematician, physicist and philosopher of science at the beginning of the 20th century. He was one of a small but honest group of scientists who refused to accept Hertz's experiments as conclusive. Moreover, Duhem was the first to raise doubts about the whole concept of crucial experiments $^{(33)}$. A good mathematician and outspoken critic of the inconsistencies in Maxwell's theory, he became one of the principle advocates of Helmholtz's approach ${ }^{(34)}$ :

...Physicists are caught in this dilemma: Abandon the traditional theory of electric and magnetic distribution, or else give up the electromagnetic theory of light. Can they not adopt a third solution? Can they not imagine a doctrine in which there would be a logical reconciliation of the old electrostatics, of the old magnetism, and of the new doctrine that electric actions are propagated in dielectrics? This doctrine exists; it is one of the finest achievements of Helmholtz; the natural prolongation of the doctrines of Poisson, Ampére, Weber and Neumann, it logically leads from the principles laid down at the beginning of the nineteenth century to the most fascinating consequences of Maxwell's theories, from the laws of Coulomb to the electromagnetic theory of light; without losing any of the recent conquests of electrical science, it re-establishes the continuity of tradition.

However, it appears that this call of Duhem's for a third solution fell mainly on deaf ears. So, whilst appreciating the difficulties of Hertz's pioneering investigations, and taking into consideration his struggle through the uncertainties and controversies of the electrodynamics of his time, the fact remains that his final opting for Maxwell's theory was not based on strict scientific logic. What needs to be done, therefore, is clearly to identify the criteria for acceptance and apply these to the existing alternative theories. The detailed examination of how and why a certain theory is confirmed or refuted by experimental tests is, of course, a matter of the methodology and philosophy of science. Hertz was apparently not fully aware of the need to test his choice from this systematic, methodological and philosophical standpoint.

As did the majority of his contemporaries, Hertz intuitively applied a criterion of empirical verification, in the hypothetico-deductive manner prevailing in the 19th century science. This method consisted of creating hypotheses in the form of postulates and then making deductions from these which could be either confirmed or rejected 
by experiment. However, from the modern methodological standpoint, as is now well recognized, empirical verification is a condition that is necessary but not sufficient for establishing the truth of a theory. The fact is that an empirical observation may verify any number of different, yet equally valid theories sharing that same prediction. The only reliable way, therefore, of deciding between theories is not to verify any one of them in particular, which might just as well verify any number of them but, if possible, to eliminate all but one of the contenders. The only way of doing this is, of course, by the method of refutation. This method may be carried out logically, by pure ratiocination (as by pointing out some logical or mathematical contradiction) or by demonstrating that empirical predictions made by some logically consistent theory are false. However, even if a theory is logically sound, if it makes no falsifiable predictions, it cannot be refuted. Such a theory, according to Popper, cannot qualify as a scientific theory. This criterion, introduced by Popper in the late 1920s, thus provided a reliable criterion for separating genuine scientific theories from pseudo-scientific or metaphysical theories by which, Popper meant theories which make no predictions that can, even in principle, be empirically falsified.

It may be of interest to consider how this modern criterion of falsifiability might have been influenced on Hertz's decision, had it been available at the time. Qualitatively, as we have seen, both Maxwell's and Helmholtz's theories fit equally well all the observations made by Hertz, namely:

1) material changes in the surrounding space;

2) finite propagation of transverse components with the velocity of light;

3) empirical observations of energy transfer etc.

The present-day scientific method suggests that the next step is to explore the difference between the experimental predictions of the two theories, beyond those that are already known, and to separate-out the different, non-compatible but empirically verifiable predictions that is, to determine which of them, if any, are sufficient to explain all known facts, as distinct from those that are merely necessary. Thus, in the case of alternative electrodynamics theories, the core of the additional 'crucial' experiment should have been to test, experimentally, statements specifically describing the character of the longitudinal electric components which distinguish the Helmholtzian from the Maxwellian theory. A clear absence of any indications on longitudinal character of interactions in all observable cases would immediately imply Maxwell's theoretical approach beyond any doubt. Since no decisive, unambiguous information of the kind necessary to refute Helmholtz's theory has yet been found in Hertz's experimental results, and, hence no sufficient criterion had been established to accept the Faraday-Maxwell field interpretation. Moreover, as we mentioned above, electrostatic interactions always act along the line joining positions of two charges at rest, i.e. they are manifestly longitudinal. Proper Maxwell's equations give the same picture in electro- and magnetostatic limits. The point is that longitudinality of interactions is an essential, irremovable feature in many observable phenomena. There was undoubtedly a real difficulty here, realized by Hertz himself after frustrated efforts to dissociate any reference to IAAAD as far as to interaction was concerned. 
The criterion on empirical observations of energy transfer comes from Faraday. He believed that any interaction should be accompanied by material changes in the surrounding space, that its propagations should take time. According to this reasoning, the alternative views would violate the conservation of energy because it would appear in the body without passing the intervening space. Later on Poynting developed this conception in deeper details, so that the energy could be seen in many respects similar to a material fluid satisfying equations of conservation and continuity. The flux of interaction was since then up till now associated with the flux of electromagnetic field energy. Nevertheless, it should be recognized that there are still various well-known difficulties in modern electrodynamics in describing the energy flow in some physical situation.

On the contrary, Helmholtz's position differentiated both kinds of energy: potential and kinetic as it was done in mechanics. The energy of transverse waves represented material kinetic energy localized in space between radiating bodies whereas potential energy had no material equivalent and did not show any property of its manifest accumulation in the intervening space (non-local and non-material property). Thus, in Helmholtz's interpretation longitudinal components (responsible for interaction) did not take part in any energy carrying through the space. Immaterial status of longitudinal instantaneous forces enable them to avoid a possible conflict with the special relativity. In fact, it is well-known that Einstein's theory does not limit phase velocities, if there is no local energy transfer.

Before long physicists ceased to ask why no scientific device is able to detect any observable energy flow between two interacting bodies at rest. Recent photon teleportation experiments also questioned actual understanding of localization of electromagnetic energy. These difficulties seem to arise in modern physics from the indispensability of material nature of all kinds of electromagnetic and gravitational interactions. This comes from Faraday and Maxwell who thought that if the IAAAD concept did not imply any material mediator, then it did not imply any conceivable physical mediator at all. Therefore, in respect to the energy, they thought it could have only material origin, being, like matter, accumulated locally and transferred continuously in space. This path of reasoning also explains the modern prejudice against immateriality, even though space and time can hardly be classified among material objects and hence cannot possess only material properties. Consideration of both kinds of electromagnetic energy following Helmholtz (local along with non-local, i.e. material kinetic along with potential) helps to overcome this fundamental methodological inconsistency but on theoretical foundations different from those of purely material Faraday-Maxwell's field theory.

On the other hand, as already mentioned, the criterion of falsifiability requires any truly scientific alternative theory to be logically self-consistent. Logical inconsistencies lead to bogus predictions. In this way it is interesting to remind that several aspects of standard conventional electrodynamics are found to be unsatisfactory, despite all the advances claimed by relativity and quantum mechanics. Conventional electrodynamics is thus still not free from untractable inconsistencies, as in its implications regarding self-interaction, infinite contribution of self-energy, the concept of electromagnetic mass, indefiniteness in the flux of electromagnetic energy, etc. These internal difficulties explain why, from the beginning to the middle of the 20th century, there were unceasing 
efforts to modify either Maxwell's equations or the underlying conceptual premises of electromagnetism. The present status of classical electrodynamics can be expressed by words of R. Feynman ${ }^{(35)}$ :

...this tremendous edifice [classical electrodynamics], which is such a beautiful success in explaining so many phenomena, ultimately falls on its face. When you follow any of our physics too far, you find that it always gets into some kind of trouble. the failure of the classical electromagnetic theory. ...Classical mechanics is a mathematically consistent theory; it just doesn't agree with experience. It is interesting though, that the classical theory of electromagnetism is an unsatisfactory theory all by itself. There are difficulties associated with the ideas of Maxwell's theory which are not solved by and not directly associated with quantum mechanics...

One of the latest systematic accounts of these difficulties has been made recently in ${ }^{(36)-(37)}$. In particular, the old problem of Maxwell's electrodynamics, concerning the uncertain relationship between static and dynamic limits, has been brought into greater relief. Pure mathematical analysis ${ }^{(36)}$ has shown that the conventional theory does not ensure a continuous transition between static and dynamic limits, which is, surely, in itself, strange to contemplate in the context of the Faraday-Maxwell continuous-field concept. Interestingly, it has also been found that if the condition of continuous transition between static and dynamic limits is imposed explicitly in mathematical terms, then conventional boundary condition should be replaced by new generalized boundary conditions. As a result, solutions of Maxwell's equations have to be also modified to include instantaneous longitudinal components. It would be of interest to note that the possible difficulty with conventional boundary conditions for field equations in the classical field theory was realized by A. Einstein himself a few month before his death in 1955. In the last edition of "The Meaning of Relativity" he added the following ${ }^{(38)}$ :

A field theory is not yet completely determined by the system of field equations. Should one postulate boundary conditions? Without such a postulate, the theory is much too vague. In my opinion the answer to the question is that postulation of boundary condition is indispensable.

Thus, a different choice of boundary conditions can result in essentially different approach (Maxwellian or Helmholtzian), provided the same system of Maxwell's equations (a limit case of Helmholtz's equations).

In summarizing this discussion it is interesting to note another possible attractiveness of Helmholtz's conceptual foundations. As well as having these above-mentioned difficulties, Maxwell's theory also could not provide any reliable model of the atom. This left a theoretical gap which had to be filled independently. This, of course, is what led to the current quantum mechanical theory of the atom, with its implications of essential non-locality. Thus was created the other serious dilemma of present-day physics: significant incommensurability between the classical relativistic theory with its basic concept 
of local material field, and quantum mechanics with its essential non-locality. This fundamental conflict is such that, for some people, the only way of resolving it seems to be to combine or superimpose these incompatible requirements for locality and non-locality in some purely expedient and compromising way. In view of these reasoning it also can be suggested that any physical theory of interaction might not be pure field theory (in the sense of local field) but be complemented by instantaneous longitudinal components (in the sense of non-local potential field).

\section{Conclusions}

Thus, as the relevant historical literature shows, Helmholtz's theoretical foundations never contradicted Hertz's experimental observations. Hertz's arguments in favor of Maxwell's theory had not been based on a solid scientific and logical grounds as it has been shown from the position of modern science and from the analysis of very recently published Hertz's laboratory notes, dairy and private communications ${ }^{(22)}$.

Nowadays, Helmholtz's conceptual foundations promise an alternative consistent solution to fundamental problems of modern physics at reconciling classical electrodynamics and quantum mechanics in a less ad hoc and altogether more rational way than has, up till now, seemed obligatory. Recent experimental confirmations of the violation of Bell's inequalities in quantum mechanical measurements, entanglement and teleportation in quantum optics shed some new light on possible alternative foundations of classical electrodynamics.

The crisis which arose in classical physics at the very end of the 19th century accounted to the limitations of classical explanation did not implied, however, radical revision of all foundations and left unquestionable many previous results: among them conceptual foundations of Maxwell's theory and Hertz's incomplete experimental approach. Many problems of classical electromagnetic research passed unnoticed in view of novel and promising perspectives opened by special relativity and quantum mechanics. Nevertheless, nowadays it is still unclear by what reasons the progress of science at the beginning of the 20th century was so unconditionally left to be dependent on formally incomplete experimental verification of fundamental issues as far as to the propagation of electromagnetic interactions is concerned.

Despite the intensive and undeniable progressive development of physics and technology since Hertz's experiments, there is, however, an uneasy feeling that we may not be on a very solid ground with respect to the nature of interactions. In fact, modern explanation of interaction process as an interchange of real and virtual particles is still incomplete, posing more fundamental questions than answers. In this respect, the standard model has very high expectation on observations of fundamental Higgs's massive particles in expensive CERN's experiments (approximately in 2005). The failure would mean a possible crash of the conventional approach. Why not to choose less expensive but not less convincing way: using modern technological advantage over Hertz's experimental facilities, it would be relatively easy to complete Hertz's empirical task definitively and find out whether the propagation of electromagnetic interactions is only 
transverse (by means of massless photons), only longitudinal (Helmholtz's non-locality) or both by nature on the classical level. This latter task can also be considered as a sufficient condition for verification of Maxwell's or Helmholtz's foundations.

\section{APPENDIX A. Foundations and Structure of Helmholtz's Theory}

The original Faraday-Maxwell form of electrodynamics which was predominant in Britain, dispensed altogether with the objects like charge in their own right. The primary entity turned out to be the field. The notion of a charge was very problematic in Maxwell's original theory that made the electromagnetic fields to be divorced from their sources. In Buchwald's words, "in Maxwellian theory, charge is produced by the electric field...The Maxwellian goal was to create a theory of electromagnetism which made no use whatsoever of the microstructure of matter" (J. Buchwald, "From Maxwell to Microphysics", The University of Chicago Press, 1985). In other words, in the original field theory the source was a secondary conception conceivable as a sort of field singularity. In modern notation, this approach leads to a particular form of continuity equation with no open currents $\mathbf{J}$ : (i.e. $\nabla \cdot \mathbf{J}=0$ ). (Later on the notion of a source in Maxwell's theory was changed by Lorentz and used in his microscopic electron theory of electromagnetism accepted nowadays). The interaction between objects was reduced to the change of state of the surrounding field. Hence, no direct interaction was admitted in Faraday-Maxwell's electrodynamics as in any other possible field theory, based on the notion of local Faraday field. This marked the main division between Maxwell's and Helmholtz's conceptions.

Helmholtz created a theory that differed radically from the Faraday-Maxwell scheme. He did not see it appropriate to use additional theoretical 'artifact', i.e. the Faraday notion of local field. On the contrary, Helmholtz accepted (following the main trend of the continental electromagnetism) the underlying electric microstructure of matter as a primary hypothesis and, hence, used a continuity equation that linked charge and currents accepted in our modern notations. On the other hand, he based his analytical approach on a notion of potential functions. Besides the notion of a static potential (nowadays known as a scalar potential function) satisfying inhomogeneous Poisson's equation, Helmholtz postulated the existence of electrodynamic potential $\mathbf{U}$ (nowadays known as a vector potential function) that generalized the conception of a potential so successfully used in magnetostatics. The source (charge or current) specified in Helmholtz's approach the nature of interaction (electric or magnetic). All objects interacted directly, all forces among them could be deduced in terms of potential functions and hence there was no intermediary local field to interact with. Another essential point to grasp about the fundamental structure of Helmholtz's electrodynamics is that every system state can be described by the energy of interaction based on both electrodynamic and static potentials. It reflects Helmholtz's conviction in the profound truth of the Principle of Least Action. (Modern field theory is also coupled to Hamilton's principle).

Helmholtz considered the total current $\mathbf{C}_{t o t}=\mathbf{C}_{c o n d}+\frac{\partial \mathbf{P}}{\partial t}$ to be formed of conduction currents $\mathbf{C}_{\text {cond }}$ and of changing dielectric polarization $\mathbf{P}$. The latter, according to the Mossotti hypothesis (1846) would implicate currents in precisely the same way that 
changing charge densities implicate conduction currents. Helmholtz linked electrodynamic potential $\mathbf{U}$ to the total current $\mathbf{C}_{t o t}$ :

$$
\mathbf{U}(\mathbf{r})=\int \frac{\mathbf{C}_{t o t}\left(\mathbf{r}^{\prime}\right)}{\mathbf{r}_{d}} d^{3} \mathbf{r}^{\prime}+\frac{1-k}{2} \nabla_{\mathbf{r}} \int\left[\mathbf{C}_{t o t}\left(\mathbf{r}^{\prime}\right) \cdot \nabla_{\mathbf{r}^{\prime}} \mathbf{r}_{d}\right] d^{3} \mathbf{r}^{\prime}
$$

where $k$ is undefined constant; $\mathbf{r}, \mathbf{r}^{\prime}$ are positions of current differential elements and $\mathbf{r}_{d}=\mathbf{r}-\mathbf{r}^{\prime}$ is the distance between them. This gives the expression for $\mathbf{U}$ to which Maxwell's, Weber's and Neumann's theories lead by setting $k$ equal, respectively, to 0 , -1 , and 1.

Dielectric polarization $\mathbf{P}$ in this approach can be created by electrodynamic potential $\mathbf{U}$ (representing magnetic forces) as well as by static potential $\Phi$ (representing electric forces):

$$
\mathbf{P}=-\chi \nabla \Phi-\chi A^{2} \frac{\partial \mathbf{U}}{\partial t}
$$

where the quantity $A$ depends on the medium susceptibility $\chi$ and, as it will be seen later, defines the propagation speed of transverse electromagnetic waves. The term $\frac{\partial \mathbf{U}}{\partial t}$ mathematically describes the interaction effect of changing currents on charges. Conversely, Helmholtz assumes that the spacial variation of the static potential $\Phi$ shows the effect of a charge in producing a current (Ohm's law):

$$
\mathbf{C}_{\text {cond }}=\frac{1}{R}\left(A^{2} \frac{\partial \mathbf{U}}{\partial t}-\nabla \Phi\right)
$$

where $R$ is the resistivity of a conductor.

Helmholtz also considered continuity equation for total currents

$$
\boldsymbol{\nabla} \cdot \mathbf{C}_{t o t}+\frac{\partial \rho}{\partial t}=0 \quad\left(\mathbf{C}_{t o t}=\mathbf{C}_{c o n d}+\frac{\partial \mathbf{P}}{\partial t}\right)
$$

and Poisson's equation for static potential

$$
\nabla^{2} \Phi=-4 \pi \rho
$$

All these equations (1)-(5) constitute the foundations of Helmholtz's electrodynamics. In this approach all forces can be deduced in terms of the potential functions $\mathbf{U}$ and $\Phi$. In other words it means that the underlying analytical structure of Helmholtz's electrodynamics can be reduced to the partial differential equations for both potentials:

$$
\begin{gathered}
\nabla^{2} \mathbf{U}=(1-k) \nabla \frac{\partial \Phi}{\partial t}-4 \pi \mathbf{C}_{t o t} \\
\boldsymbol{\nabla} \cdot \mathbf{U}=-k \frac{\partial \Phi}{\partial t}
\end{gathered}
$$

Using these equations Helmholtz obtained a general equation for propagation: 


$$
\begin{array}{r}
-\frac{A}{R} \frac{\partial^{2} \mathbf{U}}{\partial t^{2}}+\frac{\boldsymbol{\nabla}(\boldsymbol{\nabla} \cdot \mathbf{U})}{k R}-\chi A^{2} \frac{\partial^{3} \mathbf{U}}{\partial t^{3}}= \\
-\frac{1+4 \pi \chi}{4 \pi \chi} \nabla \frac{\partial}{\partial t}(\boldsymbol{\nabla} \cdot \mathbf{U})+\frac{1}{4 \pi} \boldsymbol{\nabla} \times\left(\boldsymbol{\nabla} \times \frac{\partial \mathbf{U}}{\partial t}\right)
\end{array}
$$

If one separates cases for which $\nabla \cdot \mathbf{U}_{t}=0$ (the divergence vanishes for transverse components $\mathbf{U}_{t}$ ) and $\nabla \times \mathbf{U}_{l}=0$ (the curl vanishes for longitudinal components $\mathbf{U}_{l}$ ), the general equation for $\mathbf{U}=\mathbf{U}_{t}+\mathbf{U}_{l}$ can be split in two:

$$
\begin{gathered}
\chi \frac{\partial^{2} \mathbf{U}_{t}}{\partial t^{2}}=\frac{1}{R} \frac{\partial \mathbf{U}_{t}}{\partial t}+\frac{1}{4 \pi A^{2}} \nabla^{2} \mathbf{U}_{t} \\
\chi \frac{\partial^{3} \mathbf{U}_{l}}{\partial t^{3}}=\frac{1}{k R} \nabla^{2} \mathbf{U}_{l}-\frac{1}{R} \frac{\partial^{2} \mathbf{U}_{l}}{\partial t^{2}}+\frac{1+4 \pi \chi}{4 \pi k A^{2}} \nabla^{2} \frac{\partial \mathbf{U}_{l}}{\partial t}
\end{gathered}
$$

In non-conducting medium, the resistivity $R$ is infinite and Helmholtz's equations for propagation result considerably simplified:

$$
\begin{aligned}
\frac{\partial^{2} \mathbf{U}_{t}}{\partial t^{2}} & =\frac{1}{4 \pi \chi A^{2}} \nabla^{2} \mathbf{U}_{t} \\
\frac{\partial^{2} \mathbf{U}_{l}}{\partial t^{2}} & =\frac{1+4 \pi \chi}{4 \pi k \chi A^{2}} \nabla^{2} \mathbf{U}_{l}
\end{aligned}
$$

Same wave equations can be derived for the scalar potential $\Phi=\Phi_{t}+\Phi_{l}$.

Originally, Helmholtz's theory was conceived only for polarizable material medium. In order his equations for propagation to make sense in empty space, the notion of ether should have been accepted and the ether itself should have been considered as polarizable. It was done in an attempt to understand Maxwell's field equations as a limit case of Helmholtz's theory. This assumption that the ether exists and has a non-zero susceptibility $\chi_{0}$ introduces into Helmholtz's scheme fieldlike feature similar in status to the conception of light ether in Maxwell's approach.

Among researchers in the history of classical electrodynamics there is a full consensus that to reach formally (i.e. not on a conceptual level) the Maxwell scheme from Helmholtz's theory requires two limits: $k=0$ and infinite ether susceptibility $\chi_{0}$. The former is necessary to obtain Maxwell's equations (rewritten for 4-potential) and the latter, to make the velocity of transverse waves in Helmholtz's theory equal to the velocity of light $c$. Let us follow both limits, in order to analyze the limit behavior of transverse and longitudinal waves in Helmholtz's scheme. First, all basic constants in both theories are to be made mutually related. The constant $A$ depends on ether's susceptibility $\chi_{0}$ and can be reorganized into new quantity $c^{-1}=A \sqrt{1+4 \pi \chi_{0}}$ which is inversely proportional to the velocity of light $c$ obtained in Maxwell's theory. Hence, the speed of propagation for transverse waves in Helmholtz's theory is

$$
\mathbf{v}_{t}=c \sqrt{\frac{1+4 \pi \chi_{0}}{4 \pi \chi_{0}}}
$$


To reach effectively Maxwell's wave velocity $c$, the ether susceptibility $\chi_{0}$ must be infinite in Helmholtz's wave equations. In the case of longitudinal components, the propagation rate with reorganized $A$ constant is

$$
\mathbf{v}_{l}=c \frac{1+4 \pi \chi_{0}}{\sqrt{4 \pi k \chi_{0}}}
$$

Obviously, the longitudinal wave acquires infinite velocity by setting $\chi_{0}$ to infinity independently of the value of $k$. In no way it means that the longitudinal component as a mathematically valid solution of general Helmholtz's equation for propagation, lacks any sense or disappears as Hertz assumed in his analysis of the Maxwell limit of Helmholtz's scheme. The claim that the limit $k=0$ is necessary to reach the infinite velocity of propagation came from Helmholtz himself. Perhaps, it was due to the erroneous final expression for $\mathbf{v}_{l}=c \sqrt{\frac{1+4 \pi \chi_{0}}{4 \pi k \chi_{0}}}$ given by Helmholtz in his early work (1870), as commented by Buchwald ${ }^{(14)}$.

In fact, original Maxwell's theory did not predict any longitudinal waves because basic wave equations were homogeneous (or sourceless), reflecting the form of continuity equation used by Maxwell $\left(\boldsymbol{\nabla} \cdot \mathbf{C}_{t o t}=0\right)$. Later on, in Lorentz's discussion of Maxwell's theory (1892) a moving charge as a field source had been incorporated. It marked the main trend in modification of underlying concepts in original Maxwell's theory. Primarily, this approach transformed the Maxwell continuity equation because the conception of source resulted compatible with Helmholtz's continuity equation. Thus, original Maxwellian field theory lacked many of the conceptions that many years later formed part of a unified system known and accepted nowadays as Lorentz's microscopic theory of electromagnetism.

Lorentz's modification of Maxwell's field approach provided Lienard (1898) and Wiechert (1901) with inhomogeneous wave equations which retarded solutions were found under the well-known condition (which was thought to be just experimentally verified by Hertz (1888)) that electromagnetic interactions propagate with the velocity of light. It gave a rise to the fact that longitudinal and transverse components in Lienard-Wiechert solutions propagate at the same rate. Nevertheless, in many situations Lienard-Wiechert's longitudinal components can be eliminated by means of appropriate gauge transformations that makes their status highly uncertain in the framework of modern classical electrodynamics. On the contrary, Helmholtz's scheme in Maxwellian limit offers a more consistent approach to longitudinal components: longitudinal forces are always present and constitute bipartite, instantaneous mutual interaction (i.e., they are irremovable). This scheme, contains electro- and magnetostatics as naturally valid limit case whereas in the Faraday-Maxwell theory all static and quasistatic phenomena are totally foreign to the notion of Faraday's local field.

Another point worth emphasizing here, illustrates the similarity of Helmholtz's scheme with modern field theories. Helmholtz based his approach on the notion of electrodynamic and static potentials (vector and scalar potential function in modern notation). Potential functions are essential features of all field theories independently of a particular model of the field. Thus, the novel character of Helmholtz's electrodynamics 
consists in the combination of transverse (local) and longitudinal (non-local) parts of those potential functions. In modern retrospective, it means that Helmholtz's approach is purely field scheme, sharing elements of both local and non-local field theories.

\section{APPENDIX B. The Brief Sequence of Hertz's "Crucial" Experiments}

From Hertz's published papers we know that he was looking for the effect of interference of the action that propagated along the wire with the velocity close to the velocity of light by the direct action of oscillator that might have very different rate of propagation. Hertz reasoned in the following way: $(a)$ if both actions travel at the same rate, the interference picture will be the same no matter where it is measured (no phase shift observed); (b) if propagation speeds are not equal, then the phase difference will depend on the measurement place. In the case, the direct action of the oscillator has an infinite rate of propagation, the interference picture should reflect only the periodicity of the wire action (i.e. the interference will change sign at every half wave-length of the waves in the wire).

At the end of 1887 Hertz finally undertook this task. Recently published laboratory notes $^{(22)}$, dairy and letters to parents can get access to Hertz's first understanding of his results in such form that did not appear in his printed papers. From laboratory notes we know that on November 7 Hertz succeeded in using the resonator to detect the standing electric waves in a wire connected to one of the oscillators plate. It meant he was now prepared to undertake interference experiments. Notes dated November 11-12 record Hertz's intention to construct an experiment to detect a propagation of the "direct action" of the oscillator. In these first series of experiments Hertz set the resonator's plane vertically. He could rotate the plane of the resonator about a vertical axis so that the spark gap of the resonator could point parallel to the wire (position 1 in Hertz's terminology especially sensitive to wire action) or parallel to the oscillator gap (position 2 sensitive to direct action of the oscillator whether electrostatic or electrodynamic in Helmholtz's specification).

In his published articles Hertz explained how, rotating the resonator's plane, can be observed differences in sparking for different deviations in order to determine the interference picture in different positions from the oscillator (for greater details of these experiments see, for instance, Buchwald $\left.{ }^{(14)}\right)$. On November 11 Hertz succeeded in producing a detectable interference between the direct action of the oscillator and the wire waves. The entry of his dairy notes for November 12 reads: "Set up experiments on the velocity of propagation of the electromagnetic effect. Contrary to expectations, the result is infinite propagation".

Discouraged by futile efforts to detect novel effects (finite rate of propagation), Hertz put aside experimenting. Later on for the introduction to "Electric Waves" he gave account about the first experiment:

"...Dishearted, I gave up experimenting. Some weeks passed before I began again. I reflected that it would be quite as important to find out that electric force was propagated with an infinite velocity, and that Maxwell's theory was 
false, as it would be, on the other hand, to prove that this theory was correct, provided only that the result arrived at should be definite and certain."

Five weeks later, On December 15, Hertz returned to his experiments again. As it is seen from his dairy, he decided to write a detailed paper on negative results but only after having checked and making secure of his previous results. After two days of laboratory tests, Hertz became convinced that his device was a very reliable interference detector. This confidence allowed him to start a new series of experiments performed from December 12 through 21. They were to ensure that the device was properly constructed and calibrated before setting experiments about the propagation velocity. After having finished calibrations of his device, Hertz turned his experimentation to the main goal. On December 22, he started a next series of experiments for the resonator's position 1 and 2 (see previous description). Hertz concluded from his observations that the interference remained in step with the wire wave. In other words, it confirmed that the direct action of the oscillator had immeasurably higher speed.

From the letter to his parents written next morning, we get Hertz's first perception of this result ${ }^{(14)}$ :

"What is the unexpected and to me displeasing result of my endeavors? The velocity [of the direct action] is not that of light, but certainly much greater, perhaps infinitely great, at all events not measurable. Even if it were three times as great, it could still be measured... Now, there is no arguing with nature; it must be as it is, but I should have certainly liked it better to obtain a clear, positive result than this more negative one... Certainly, caution is indicated here, but once again the experiments seem all too clear to me."

On this same day later, December 23, $1887 \mathrm{Hertz}$ put a resonator in a different orientation. The resonator's plane was now horizontal (position 3 in Hertz's classification). After Christmas, On December 26 Hertz began a new series of experiments using the position 3 with the resonator's gap parallel to the wire. In Hertz's mind, the resonator was governed entirely by the oscillator's electrodynamic action (not electrostatic in Helmholtz's classification). According to the measured interference phase shift along the wire, Hertz unexpectedly found that the oscillator's direct action propagated 1.5 times faster than the wire wave (i.e. close to the velocity of light). Having done these promising measurements, Hertz sought a clear possibility to establish a relation between the speeds of the two actions: the electrostatic and the electrodynamics in Helmholtz's classification.

To adapt experiments for this aim Hertz used the position 3 but with the resonator's gap facing the oscillator. In order to measure interference only between two actions, Hertz for the first time removed the wire used for previous interference experiments. In this configuration the resonator responds to both static and dynamic actions, though the latter predominates. If the resonator gap faces the oscillator, the signed value of the total driving force is a positive contribution of both parts of direct action. If the 
gap faces in opposite direction $\left(180^{\circ}\right.$ rotation about the center of the resonator $)$, the contribution of the electrostatic action will change the sign. By setting the resonator in different positions Hertz obtained interference marks which he put in the final table (discussed in the main text concerning singularities in the near zone). These were Hertz's first measures of the direct action propagation without the support of the wire wave of known length. On December 30, Hertz undertook new observation up to 12 meters.

On January 1, on the first day of 1888 Hertz wrote to his parents that he was somewhat depressed, anticipating the distasteful risk of carrying on with additional experiments, "because one can only lose by them". Some days later he began writing up the article, troubled by doubts that the results might be "figments of the imagination"(14). Finally, Hertz calmed his doubts and by the end of January wrote the paper entitled, "On the Finite Velocity of Propagation of Electromagnetic Action".

The history of physics researchers seemed to show no interest to the fact that Hetrz's drastic change in attitude towards his previous "negative" results was not supported by additional reliable experimental measurements. The confirmation of this change is clearly seen in Hertz's Introduction to "Electric Waves" where he was forced to admit many doubts on the reliability and accuracy of his first negative experiments.

Nevertheless, in modern retrospective the second series of Hertz's experiments and the table obtained on December 22 (where he confirmed his previous "negative" results) do not seem to support very strongly Hertz's allegations on inaccuracy. This is also Buchwald's opinion in his own words ${ }^{(14)}$ :

...Neither the 3.4 we find in Hertz's undiscussed average not the 2.9 obtained directly from his table easily sustains a positive argument for finite propagation. On the contrary, the 2.9 result falls right between the 2.66 and 3.1 values for the wire wave's half-length that he previously found, and the 3.4 result barely misses the first, and includes the second, value if we allow the inaccuracy to be no larger than the standard deviation among the measurements. If, as Hertz would certainly have had to admit, the inaccuracies are in fact somewhat larger than this, then his numbers cannot be instrumentally distinguished from one another. These results, as they stand, accordingly nicely sustain Hertz's anticipated negative outcome. But because Hertz later decided his experiments had positive results, in his published papers he had to argue the apparent implication of the table away, which he did in two ways: first, by saying that the alternation is not at all precise (which might, however, speak just as well to the experiment's accuracy as to its implications) and, second, by capitalizing on what might otherwise appear to be inaccuracy in observation, namely, the frequent appearance of four or more zero points together at distances past $3 \mathrm{~m}$...

Thus, it is not still clear why Hertz did not overcome " the distasteful risk of carrying on with additional experiments" and did not repeat his first experiments in order to remove or confirm his doubts in their inaccuracy. His published papers, laboratory notes and dairy, unfortunately, provide little help here. 


\section{REFERENCES}

1. S. Laplace, Mecanique Celeste, Book X, 22, (Paris, 1799)

2. T. Van Flandern and J.-P. Vigier, Foundations of Physics, 32(7), 1031-1068 (2002)

3. I. Newton, Third Letter to Bentley, from Work of Richard Bentley, III, 211

4. S. Laplace, Exposition du System du Monde, Book IV (Paris, 1796)

5. P. Graneau and N. Graneau, Newton versus Einstein: How Matter Interacts with Matter (Carlton Press, New York, 1993)

6. D. Hoffmann, "Heinrich Hertz and the Berlin School of Physics", in Heinrich Hertz: Classical Physicist, Modern Philosopher, eds. D. Baird, R.I.G. Hughes and A. Nordmann, (Kluwer Academic, Dordrecht, 1998), 1-8

7. M. Heidelberger, "From Helmholtz's Philosophy of Science to Hertz's PictureTheory", in Heinrich Hertz: Classical Physicist, Modern Philosopher, eds. D. Baird, R.I.G. Hughes and A. Nordmann, (Kluwer Academic, Dordrecht, 1998), 9-24

8. J. Maxwell, On Faraday's Line of Force, Scientific Papers, Vol.1, 160 (1864)

9. M. Hesse, "Action at a Distance in Classical Physics" ISIS 46, 337-353 (1955)

10. H. Hertz, "On the Fundamental Equations of Electromagnetics for Bodies at Rest", in Electric Waves, Collection of Scientific Papers (Dover, New York, 1962), 195

11. M. Hesse, Forces and Fields: The Concept of Action at a Distance in the History of Physics (Thomas Nelson and Sons Ltd., London, 1961)

12. H. Helmholtz, Wissenschaftliche Abhandlungen, Vol. 1, (Barth, 1882), 611-628

13. P.S. Kudryavtzev, History of Physics, vol. 2, (Moscow University Press, Moscow, 1956), 206-213 (in Russian)

14. J. Buchwald, The Creation of Scientific Effects: Heinrich Hertz and Electric Waves (The University of Chicago Press, Chicago, 1994)

15. A.E. Woodruff, "The Contribution of Hermann von Helmholtz to Electrodynamics" ISIS 59, 300-311 (1968)

16. H. Helmholtz, Wissenschaftliche Abhanlugen, vol.1 (Barth, 1882), 556

17. H. Hertz, "On the Finite Velocity of Propagation of Electromagnetic Actions" in Electric Waves, 110 (1888)

18. ibid., "On Very Rapid Electrical Oscillations" in Electric Waves, 29-53 (1887) 
19. ibid., "On the Finite Velocity of Propagation of Electromagnetic Actions" in Electric Waves, 108 (1888)

20. ibid., 121

21. J. Cazenobe, Archives Internationale d'Histoire des Sciences, 32, 236-265 (1982)

22. M. Doncel, "Heinrich Hertz's Laboratory Notes of 1887" Archive for History of Exact Sciences, 49, 197-270 (1995)

23. M. Doncel, "On Hertz's Conceptual Conversion: From Wire Waves to Air Waves" in Heinrich Hertz: Classical Physicist, Modern Philosopher, eds. D. Baird, R.I.G. Hughes and A. Nordmann (Kluwer Academic, Dordrecht, 1998), 73-87

24. J. Z. Buchwald, "Electrodynamics in Context: Object States, Laboratory Practice and Anti-Romanticism" in Hermann von Helmholtz and the Foundations of Nineteenth-Century Science, Edited by D. Caham (University of California Press, Berkely, 1993), 345-368

25. H. Hertz, Electric Waves, Collection of Scientific Papers, (Dover, New York, 1962), 15

26. ibid., "The Forces of Electric Oscillations, Treated According to Maxwell's Theory" in Electric Waves, 137 (1889)

27. ibid., 151-152

28. W. Panofsky and M. Phillips, Classical Electrodynamics and Magnetism (Addison Wesley P.C., Massachusetts, Second Edition, 1962), 259-260

29. H. Hertz, "The Forces of Electric Oscillations, Treated According to Maxwell's Theory" in Electric Waves, 149 (1889)

30. ibid., "On the Mechanical Action of Electric Waves in Wires" in Electric Waves, $187(1891)$

31. M. Heidelberger, "From Helmholtz's Philosophy of Science to Hertz's PictureTheory" in Heinrich Hertz: Classical Physicist, Modern Philosopher, eds. D. Baird, R.I.G. Hughes and A. Nordmann (Kluwer Academic, Dordrecht, 1998), 18

32. H. Hertz, Electric Waves, Introduction, 18-19

33. P. Duhem, The Aim and Structure of Physical Theory (Princeton University Press, Princeton, 1954)

34. P. Duhem, Les Theories Electriques de J. Clerk Maxwell, Paris, 1902 (quoted from A. O'Rahilly, Electromagnetic Theory: A Critical Examination of Fundamentals, vol. 1 (Dover, New York, 1965), 161-180) 
35. R.P. Feynman, Lectures on Physics: Mainly Electromagnetism and Matter (AddisonWesley, 1964)

36. A. Chubykalo and R. Smirnov-Rueda, Physical Review E, 53, 5373-5381 (1996)

37. A. Chubykalo and R. Smirnov-Rueda, Modern Physics Letters A, 12(1), 1-24 (1997)

38. A. Einstein, The Meaning of Relativity, (Princeton University Press, Princeton, 5th Edition, 1955) 